\title{
NOUVELLE
}

\section{Connexines, rénine et hypertension}

Jacques-Antoine Haefliger, Paolo Meda
> Le fonctionnement des organismes multicellulaires repose sur un système complexe de communication, permettant aux cellules d'interagir de manière coordonnée entre elles et avec leur environnement. Cette organisation repose, entre autres, sur un mode de communication direct, les jonctions communicantes appelées jonctions gap. Ce sont des canaux intercellulaires mettant en contact le cytoplasme de deux cellules voisines. Un canal est constitué par l'assemblage de deux demi-canaux appelés connexons, fournis chacun par l'une des deux cellules adjacentes. Chaque connexon résulte de l'association de six protéines, les connexines (Cxs), qui s'assemblent en délimitant un pore hydrophile. Les connexines constituent une famille multigénique dont les membres présentent une forte homologie, sauf au niveau de la boucle intracellulaire et de la terminaison carboxyle, qui diffèrent en longueur et séquence d'une connexine à l'autre. Ces variations confèrent aux jonctions communicantes des propriétés de conductance et de perméabilité différentes selon les connexines qui les composent. Elles permettent aussi de classer les connexines selon une nomenclature dérivée de leur poids moléculaire (de la plus petite de $23 \mathrm{kDa}$ ou Cx23, à la plus grande de 62

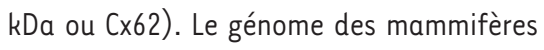
contient 21 gènes codant une connexine. Toutes les connexines ont une séquence, une structure tertiaire et une topographie intramembranaire similaires [1]. Au niveau des jonctions communicantes, les connexines de deux cellules s'apparient pour former des canaux membranaires qui mettent en communication le cytoplasme d'une cellule avec celui de la cellule adjacente, ce qui permet des échanges intercellulaires d'ions et de petites molécules cytosoliques [2]. Selon le type de connexine, ce passage est plus ou moins important et sélectif. Par exemple, les canaux formés par la Cx43 ont des propriétés très différentes de ceux formés par la $C \times 32[1,3]$. La présence des connexines dans quasiment tous les tissus de tous les organismes vivants suggère un rôle fondamental dans la coordination de populations multicellulaires. L'analyse de différents modèles cellulaires et animaux a en effet montré que la mutation, la suppression ou la surexpression de connexines spécifiques modifient de nombreuses fonctions cellulaires, y compris la sécrétion de diverses hormones [4]. L'importance de cette régulation est suggérée par l'identification d'un nombre croissant de maladies humaines dues à des connexines [5].

En clinique, les causes de l'hypertension artérielle restent obscures dans la plupart des cas. Des études récentes indiquent que les connexines sont importantes dans le contrôle de la tension artérielle au niveau de l'appareil juxta-glomérulaire du rein, un ensemble de divers types cellulaires qui interagissent les uns avec les autres pour évaluer la concentration de $\mathrm{Na}^{+}$, ainsi que le débit et la pression sanguine, dans le cortex rénal. En fonction de ces paramètres, l'appareil juxta-glomérulaire règle la sécrétion de rénine, une hormone qui, en activant le système angiotensine, régule la pression sanguine $[6,7]$. Cela implique une coordination précise des cellules de l'artériole afférente de l'appareil juxtaglomérulaire qui, in situ, sont couplées par les $\mathrm{C} \times 40, \mathrm{C} \times 37$ et $\mathrm{C} \times 43$ [8]. Pour évaluer l'influence de ces protéines dans le contrôle de la tension artérielle, nous avons pratiqué chez des rongeurs un rétrécissement chirurgical d'une artère rénale qui, en diminuant la perfusion du rein, entraîne une augmentation de la sécrétion de rénine et une hypertension analogues à celles observées chez des patients présentant une sténose de l'artère rénale. À terme, les animaux chroniquement hypertendus développent, comme les patients, une hypertrophie compensatoire du myocarde. Nous avons observé une augmentation des $\mathrm{C} \times 40$ et $\mathrm{C} \times 43$ dans la paroi aortique des animaux hypertendus [6, $9,10]$. Partant de l'hypothèse que la $C x 43$ est indispensable pour adapter la paroi artérielle aux conditions hémodynamiques imposées par l'hypertension [8], nous avons étudié des souris transgéniques (KI32) dans lesquelles la $C \times 43$ est remplacée par la $C \times 32$ [3]. Le promoteur natif de la Cx43 étant préservé chez ces souris, l'expression de la Cx32 s'y fait en lieu et place de la Cx43 native, notamment au niveau des vaisseaux du cortex rénal et du myocarde (Figure 1). Les animaux homozygotes, chez lesquels ce remplacement est complet, ont montré une tension artérielle normale et comparable à celle de la fratrie hétérozygote, coexprimant la $\mathrm{Cx} 43$ et la $\mathrm{Cx} 32$, et de la fratrie sauvage, n'exprimant pas la $C \times 32$ au niveau du système cardio-vasculaire (Figure 1). Après rétrécissement d'une artère rénale, les animaux sauvages et hétérozygotes sont devenus hypertendus et ont montré l'augmentation attendue de la rénine circulante et du volume du myocarde. Étonnamment, la fratrie homozygote maintient des taux de rénine plasmatiques bas qui permettent à ces animaux de rester normotendus [3]. 


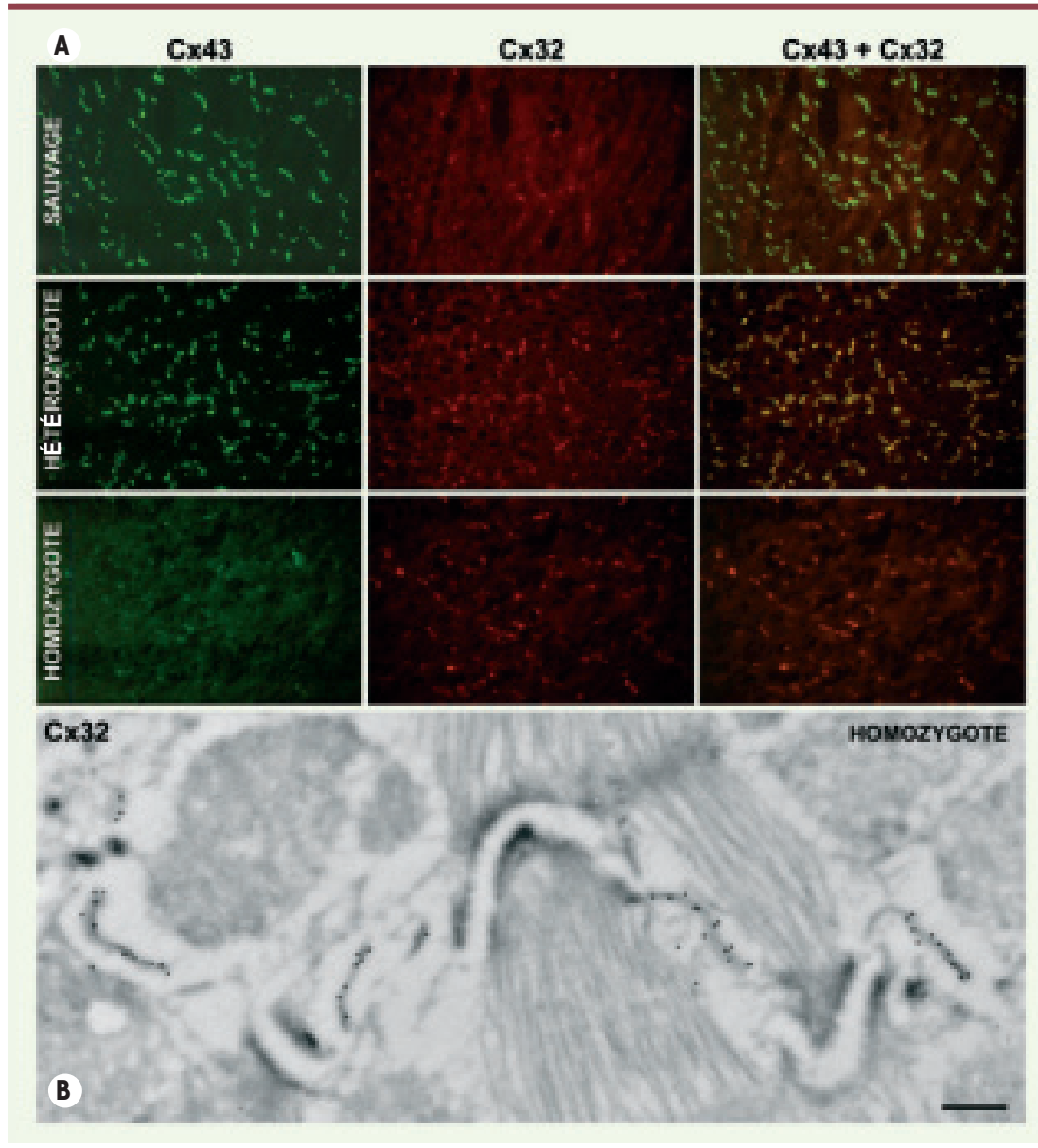

Figure 1. La Cx32 remplace la Cx43 dans le myocarde des souris KI32. A. L'immunofluorescence révèle la $\mathrm{C} x 43$ au niveau des jonctions gap du myocarde des souris de type sauvage. Cette protéine est colocalisée avec la $\mathrm{C} \times 32$ dans le myocarde de la fratrie hétérozygote. Chez les animaux homozygotes, seule la Cx32 est exprimée au niveau des traits scalariformes. $\boldsymbol{B}$. La microscopie électronique confirme qu'au niveau de ces structures, la $\mathrm{C} \times 32$ est restreinte aux jonctions de type gap. Barre de grossissement : $50 \mu \mathrm{m}$ (A) et $250 \mathrm{~nm}$ (B).

Le mécanisme responsable de cet effet doit encore être élucidé. L'hypothèse est que le remplacement de la Cx43 par la $\mathrm{Cx} 32$ perturbe la perception par les cellules endothéliales du rein des changements de pression artérielle engendrés dans le rein sténosé. La Cx32, ayant des propriétés différentes de la $\mathrm{C} \times 43$, ne peut pas trans- mettre les informations chargées d'activer les mécanismes assurant la stimulation de la sécrétion de la rénine [4].

En montrant l'implication des connexines dans le fonctionnement des cellules à rénine, ces travaux élucident l'un des mécanismes qui prédispose à l'hypertension artérielle et à ses conséquences chroniques. Ils laissent espérer que des agents pharmacologiques agissant sur les connexines pourraient permettre une prise en charge plus ciblée de cette affection courante. $\diamond$

Connexins, renin and hypertension

\section{RÉFÉRENCES}

1. Sohl G, Willecke K. Gap junctions and the connexin protein family. Cardiovasc Res $2004 ; 62$ : 228-32.

2. Meda P. Connexines, canaux jonctionnels et communications cellulaires. Med Sci (Paris) 1996 ; $12: 909-20$.

3. Haefliger JA, Krattinger N, Martin D, et al. Connexin43dependent mechanism modulates renin secretion and hypertension. J Clin Invest 2006 ; 116 : 405-13.

4. Michon L, Nlend Nlend R, Bavamian S, et al. Involvement of gap junctional communication in secretion. Biochim Biophys Acta 2005 ; 1719 : 82-101

5. Allagnat F, Krattinger N, Nicod P, et al. Gap functions and diseases. Rev Med Suisse $2005 ; 1: 1126-32$.

6. Haefliger JA, Demotz S, Braissant 0 , et al. Connexins 40 and 43 are differentially regulated within the kidneys of rats with renovascular hypertension. Kidney Int $2001 ; 60$ : 190-201.

7. Zhang J, Hill CE. Differential connexin expression in preglomerular and postglomerular vasculature: accentuation during diabetes. Kidney Int 2005; 68 : 1171-85.

8. Haefliger JA, Nicod P, Meda P. Contribution of connexins to the function of the vascular wall. Cardiovasc Res 2004 ; 62 : 345-56.

9. Haefliger JA, Castillo $\varepsilon$, Waeber $\mathrm{G}$, et al. Hypertension increases connexin 43 in a tissue-specific manner. Circulation 1997 ; 95 : 1007-1014.

10. Haefliger JA, Meda P, Formenton A, et al. Aortic connexin 43 is decreased during hypertension induced by inhibition of nitric oxide synthase, Arterioscler Thromb Vasc Biol 1999 ; 19 : 1615-22

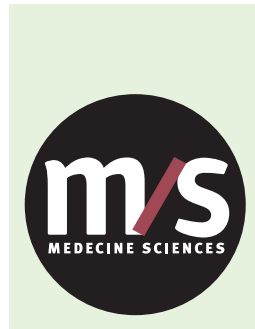

Tarifs d'abonnement M/S - 2006

Abonnez-vous

à Médecine/Sciences
> Depuis 20 ans, grâce à $m / s$, vous vivez en direct les progrès des sciences biologiques et médicales

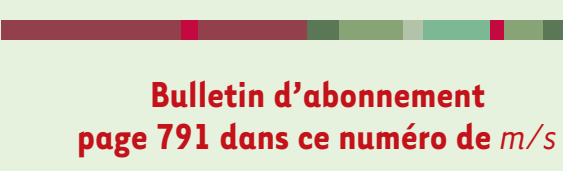

